\title{
Non-invasive pulsed Doppler study of mitral stenosis and mitral regurgitation: preliminary study
}

\author{
B. DIEBOLD, P. THEROUX, M. G. BOURASSA, C. THUILLEZ, \\ P. PERONNEAU', J. L. GUERMONPREZ, M. XHAARD, AND D. D. WATERS
}

From the Department of Medicine, Montreal Heart Institute, University of Montreal Medical School, Montreal, Canada; and Clinique Cardiologique and ERA, Centre National de la Recherche Scientifique, Hôpital Broussais, Paris, France

SUMmaRY The potential value of pulsed Doppler echocardiography in the evaluation of mitral valve lesions was studied before cardiac catheterisation in 64 patients with a clinical diagnosis of mitral valve disease. Mitral stenosis was evaluated with the transducer sited at the apex and oriented towards the mitral orifice. Reproducible tracings were obtained when a mitral flow running towards the transducer was recorded when the Doppler sample volume was located close to the mitral annulus and when the flow curves ending with the QRS complex of the electrocardiogram were retained for analysis. Of 37 patients in sinus rhythm, the 14 without a mitral valve gradient had a bifid diastolic flow pattern. In patients with mitral valve stenosis, 3 distinct flow patterns corresponding to significantly different mitral valve gradients were defined. In the presence of atrial fibrillation the Doppler analysis could not differentiate patients with mild mitral stenosis from those without a mitral valve gradient. With moderate and severe stenosis, however, the flow curves were similar to those in sinus rhythm and the method was reliable.

Mitral regurgitation was studied by 2 methods. The first was previously described for the study of mitral stenosis and was later discarded because of low specificity and sensitivity. Use of a parasternal approach improved the performance and the Doppler results were in good agreement with the left ventriculogram when significant mitral regurgitation was present. Thus, these preliminary results suggest that pulsed Doppler echocardiography may be useful as a clinical adjunct for assessing the presence and severity of mitral valve disease.

M-mode echocardiography is frequently used for studying normal and abnormal mitral valve motion. Its usefulness and limitations have been well defined (Feigenbaum, 1976). Bidimensional echocardiography allows a more complete delineation of structures and is currently under study (Henry et al., 1975). Pulsed Doppler echocardiography permits the study of blood flow velocities and profiles (Péronneau and Léger, 1969; Baker, 1970) and, therefore, may be better suited to the investigation of dynamic function. The potential value of this noninvasive approach for evaluating mitral valve stenosis and regurgitation was assessed in this study by correlating the Doppler flow signals to haemodynamic data.

'Present address: Recherche à l'INSERM, France.

Received for publication 19 December 1978

\section{Methods}

I: DOPPLER PRINCIPLE AND

INSTRUMENTATION

The directional range-gated pulsed Doppler instrument used in this study was a prototype developed by ERA, CNRS 07-785, France. An improved unit is now commercially available (Alvar Electronic). According to the Doppler principle, ultrasound waves are backscattered with a shifted frequency by moving erythrocytes. The difference in frequency is called the Doppler shift and is proportional to the velocity of the target erythrocytes and to the angle between the emitted ultrasound beam and the direction of moving blood cells. When the angle is close to zero, the Doppler shift of frequency is maximal; this frequency shift decreases progressively as the angle moves towards $90^{\circ}$ where it becomes absent. With a wider angle the flow curve 
A

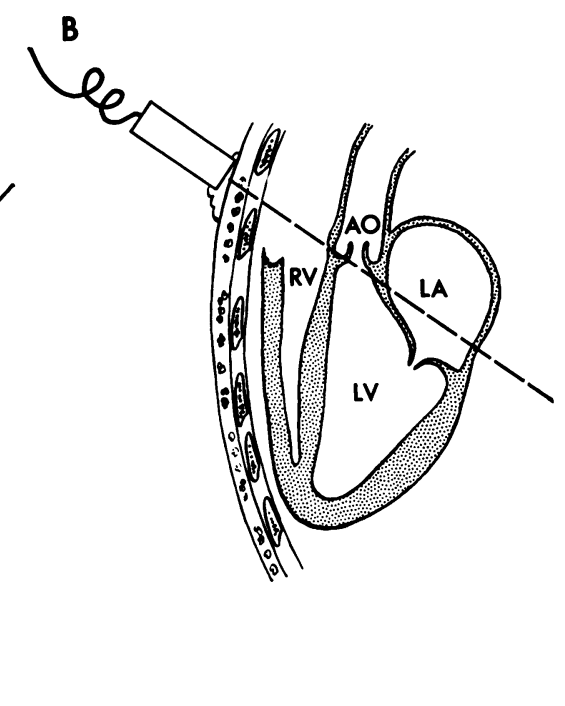

Fig. 1 Schematic diagrams illustrating the methods of examination. In panel A, the transducer is positioned at the apex and oriented towards the mitral valve in the axis of mitral valve flow. The angle between the ultrasound beam and the blood flow is close to zero and the Doppler shift is near maximal. In panel B, the transducer is located in the parasternal position and the left atrium is explored by moving the sample volume above and behind the mitral annulus. The apical approach was used in the study of mitral valve stenosis. For mitral valve regurgitation the parasternal approach gave the best results. The dotted line indicates the beam of ultrasound. The sample volume can be sited anywhere along this beam. $R V$, right ventricle; Ao, aorta; $L A$, left atrium; $L V$, left ventricle.

becomes inverted. The analogue curve of velocity was derived from a zero crossing detector and transcribed on a Gould strip-chart recorder model 2400 along with the electrocardiogram at a paper speed of 25 and $50 \mathrm{~mm} / \mathrm{s}$. Zero baseline flow was determined electronically. By convention, the flow curve was recorded as positive when the flow was running towards the transducer and as negative when it was running away from the transducer. In the present study we did not measure the angle between the ultrasound beam and the blood stream and did not attempt a quantification of flow.

The unit has a range-gated system which is adjustable in depth and in width. It transmits at a frequency of $4 \mathrm{MHz}$ and at a pulse repetition rate adjustable from 5 to $20 \mathrm{kHz}$. The duration of the acoustic pulse generated by the piezoelectric crystal varies from 2 to $4 \mu \mathrm{s}$. After a delay, the crystal is allowed to receive for $100 \mu$ s or more. The depth at which the velocity is detected is controlled by the duration of the delay in the receiver. It can be set from 3 to $15 \mathrm{~cm}$ depending on the pulse repetition rate, a lower pulse repetition rate being necessary for increasing depth. At the same time the pulse repetition rate has to be at least twice the expected Doppler shift. The value of the velocity measured is the average of all the velocities present in the sample volume. A high-pass filter with a cutoff frequency of $500 \mathrm{~Hz}$ eliminates low frequencies from cardiac and vessel wall motion. The site of the sample volume is displayed on the simultaneous $M$-mode echocardiogram and therefore can be exactly located and selected. This property of the pulsed Doppler technique allows a discrimination in depth and an estimation of the width of the flow.

\section{II: MATERIAL, METHODS, AND PATTERNS OF MITRAL VALVE FLOW}

The 64 patients included in this study were referred for cardiac catheterisation because of suspected mitral valve disease and were selected on the basis of good-quality $\mathrm{M}$-mode echocardiographic recordings. Fifty patients had rheumatic heart disease, 4 atherosclerotic heart disease, 5 mitral valve prolapse, and 5 cardiac disease of undetermined aetiology. Mean age was 47 years with a range of 19 to 68 . The Doppler study was done on the day before cardiac catheterisation. Routine right and left heart 
catheterisation was performed in the fasting state under local anaesthesia after premedication with diazepam $10 \mathrm{mg}$ per os. Pulmonary wedge pressure and left ventricular pressures were obtained via $8 \mathrm{~F}$ catheters connected to P23 Db Statham strain gauge transducers. They were recorded simultaneously on a Honeywell fibreoptic strip-chart recorder at paper speed $100 \mathrm{~mm} / \mathrm{s}$ and at a gain of $4 \mathrm{mmHg} / \mathrm{cm}$. Cardiac output was obtained by the Fick or the dye dilution curve method. Mean mitral valve gradient was measured by planimetry of 5 of 10 cardiac cycles. After pressure recordings, a left ventriculogram was obtained in the $30^{\circ}$ or $45^{\circ}$ right anterior oblique position. The regurgitant flow was semiquantified into 4 grades (McLaughlin and Morch, 1971); grade $0=$ absence of regurgitation when no contrast material entered the left atrium; grade $1=$ small puffs of contrast material passed into the left atrium, but in a volume insufficient to opacify the chamber completely; grade $2=$ the volume of contrast material entering the left atrium gradually built up to produce complete opacification; grade $3=$ complete opacification of the left atrium occurred in the first few cycles after injection of contrast material.

Sensitivity was calculated as the percentage of mitral lesions correctly detected by the Doppler analysis and specificity as the percentage of normal mitral valves correctly diagnosed.

Statistical analysis for mitral stenosis was performed by the Student's $t$ test. The Fisher exact probability test was used to compare the groups with mitral regurgitation.

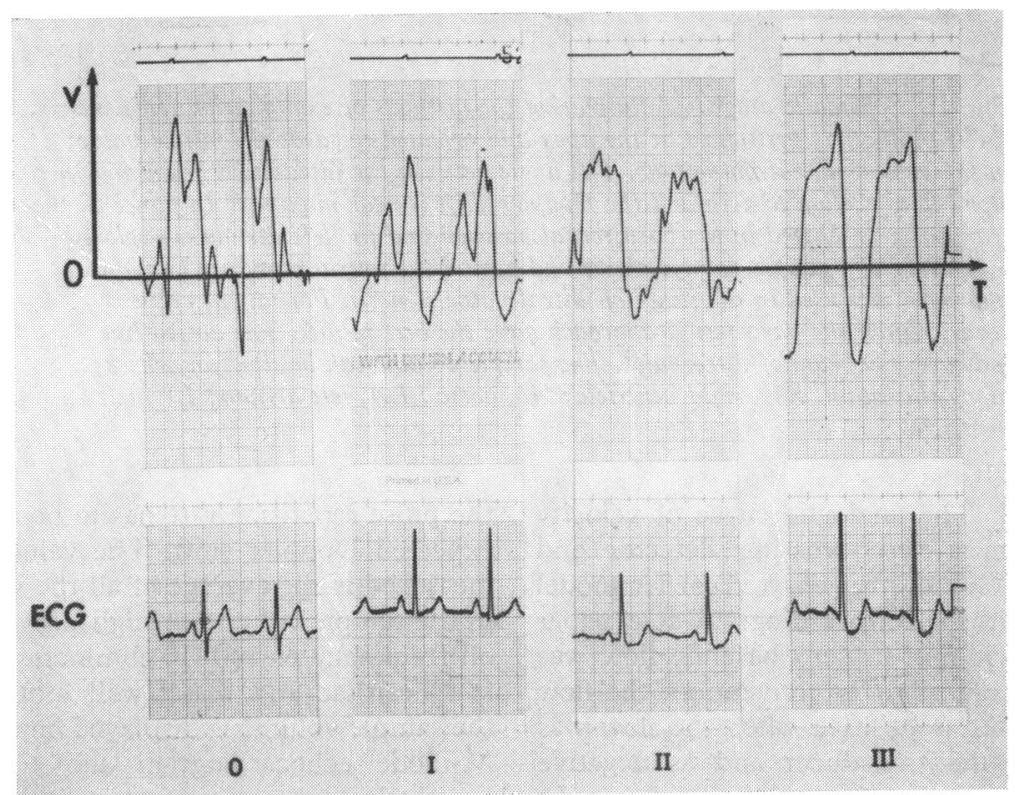

Fig. 2 Illustrative examples of mitral valve flow recordings in patients with sinus rhythm. The transducer was positioned at the apex and oriented towards the mitral valve. Four recordings are shown corresponding to the Doppler classification of mitral stenosis used in this study. Pattern 0 is a normal bifid flow and was not associated with a mitral valve gradient. Pattern I is also a two-peaked flow but with a relative increase in the amplitude of the late diastolic peak. Pattern II is a single-peaked flow curve, dome-shaped in appearance. Pattern III shows an ascending limb followed by a slight ascending plateau and a sharp decrease towards baseline at the time of the $Q R S$ complex. The examples show the recording of a negative flow during systole. This is particularly clear in pattern III. This negative flow represents a flow running away from the transducer and might originate from a regurgitant flow through the mitral valve. However, problems exist for the identification of mitral regurgitation when the transducer is located at the apex. As discussed in the text, this flow might also correspond to the ejection flow in the left ventricular outflow tract during systole. Thus, it is not specific for mitral regurgitation. $V$, velocity of flow; 0 , zero flow baseline; ECG, electrocardiogram. 


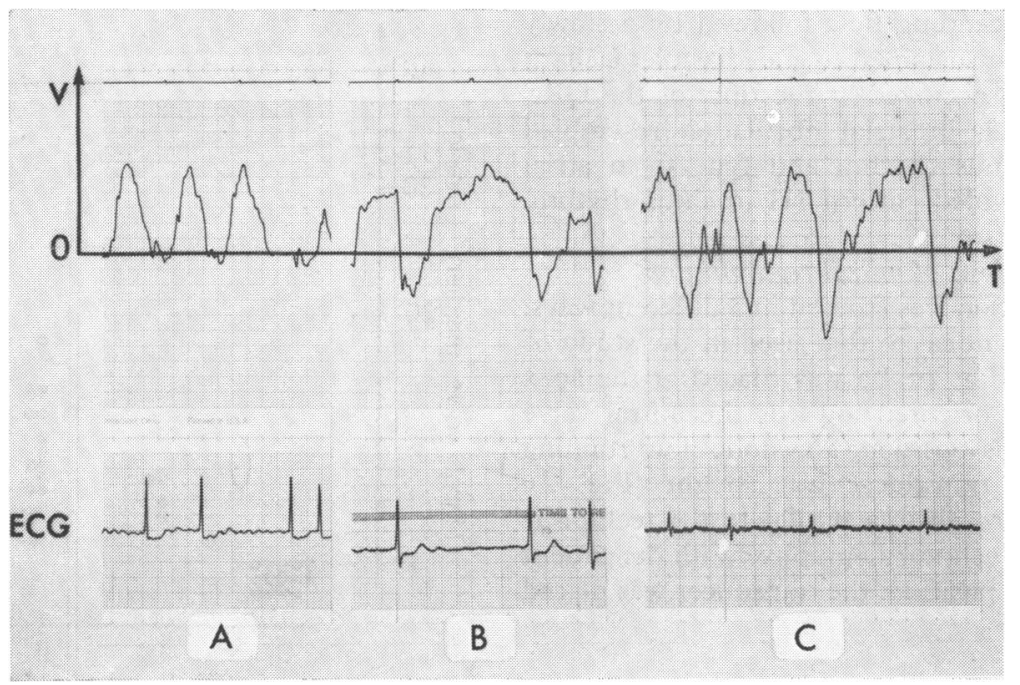

Fig. 3 Recordings of mitral valve flow in patients with atrial fibrillation. Variation in cardiac cycle length and loss of atrial contraction led to a classification based on the study of long diastoles. Type $A$ shows a flow ending before enddiastole of long cardiac cycles. Type $B$ shows a dome-shaped flow curve and type $C$ a flow with an ascending plateau throughout long diastoles. Abbreviations as in Fig. 2.

(A) Doppler analysis of mitral stenosis

The diastolic mitral valve flow was best recorded when the patients were examined in the left lateral position, the transducer being located at the site of the apical bulge and oriented posteriorly and to the right (Fig. 1A). In this position, flow from the mitral valve was in the axis of the ultrasound beam $\pm 20^{\circ}$ and was recorded as positive. The Doppler sample volume was then positioned as close as possible to the mitral annulus. A $5-\mathrm{kHz}$ pulse repetition rate was usually used. Slight adjustment of the angle of the probe and of the sample volume allowed minimisation of noise from the valve leaflets and high-quality flow curve recordings. A good and stable signal was usually recorded when the sample volume was located in the left atrium just behind the anterior leaflet of the mitral valve in its closed position. The Doppler apparatus used in this study introduced a delay of $70 \pm 10 \mathrm{~ms}$ in its process of analysing the signal. The tracings were reproducible when 3 criteria were met: (1) recording a mitral valve flow running towards the transducer in the axis of the ultrasound beam; (2) the Doppler sample volume was close to the mitral annulus; (3) the flow curves analysed ended with the $\mathbf{R}$ wave of the electrocardiogram.

Of the 64 patients, 37 were in sinus rhythm and 27 in atrial fibrillation. In patients with sinus rhythm, 4 characteristic flow patterns could be identified. They are illustrated in Fig. 2. Pattern 0 was a normal smooth flow curve with 2 conspicuons peaks, the first corresponding to rapid filling and occurring in the first third of diastole; the second peak was at the time of atrial contraction and of the A wave of the anterior mitral valve leaflet on the echocardiogram and occurred in the last third of diastole. This flow pattern was similar to the motion of the anterior leaflet of the mitral valve on $\mathrm{M}$-mode echocardiography. Pattern I was similar, but with the late diastolic peak higher than the protodiastolic peak. It was also heard as a turbulent flow. Pattern II was a single-peaked flow with a dome-shaped appearance. A low-amplitude peak of short duration was sometimes observed late in diastole but seemed to represent an artefact related to valve motion.

Pattern III was a flow with a pronounced ascending limb in the first third of diastole followed by an ascending plateau sustained until enddiastole where a sharp descending limb occurred.

In atrial fibrillation, loss of atrial contraction and variations in cardiac cycle length necessitated another classification. This classification was based on the analysis of long diastolic cardiac cycles (Fig. 3). The normal pattern (A) was a flow ending before the QRS complex of the electrocardiogram. Type B was a flow maintained throughout long diastoles, peaking in mid-diastole and thereafter being somewhat dome-shaped. Type $\mathrm{C}$ showed a 
protodiastolic ascending flow followed by a slowly ascending plateau sustained to end-diastole; a sharp descending limb occurred at the time of the QRS complex. Type B in atrial fibrillation resembled pattern II in sinus rhythm and type $C$ in atrial fibrillation resembled pattern III in sinus rhythm.

\section{(B) Doppler analysis of mitral regurgitation}

Mitral regurgitation was studied in 2 different ways. The first was identical to that used in the study of mitral stenosis. The probe was placed at the apex of the heart and oriented towards the mitral valve (Fig. 1A). In this position, a systolic flow running away from the transducer was sought (Fig. 2). The second approach was similar to the technique used in standard echocardiography. With the patient in the left lateral position, the transducer was placed

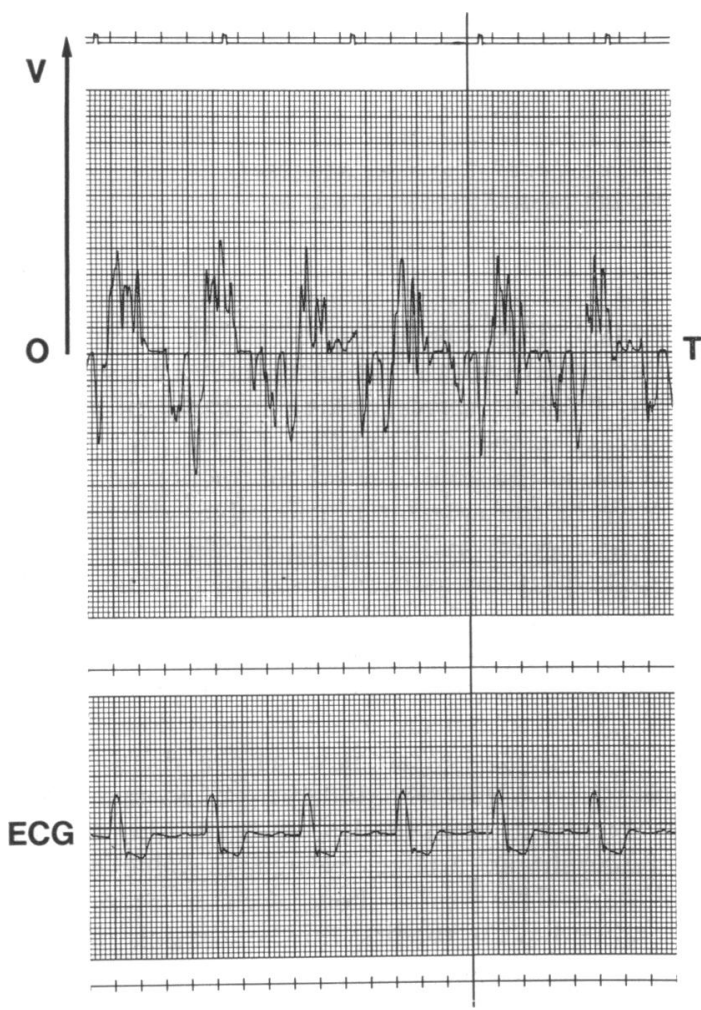

Fig. 4 Example of recording of mitral regurgitation by the parasternal approach. In this position the sample volume located in the left atrium does not override the inflow and outflow tract of the left ventricle and a systolic ejection flow cannot be recorded. The systolic flow running towards the transducer and recorded as positive represents flow from mitral regurgitation. The normal diastolic two-peaked flow through the mitral valve is also recognised running away from the transducer and recorded as negative. Abbreviations as in Fig. 2.

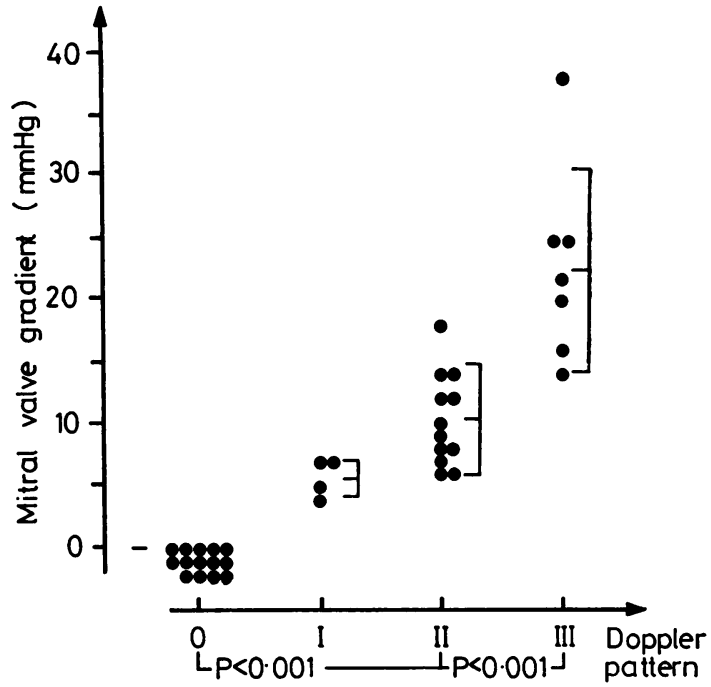

Fig. 5 The mean mitral valve gradient is plotted against the Doppler flow pattern in patients with sinus rhythm. The Doppler flow pattern could identify all cases of mitral stenosis with no false po.itives. It also allows semiquantification of the degree of stenosis. The pattern was not altered by the presence or the absence of associated regurgitation.

parasternally in the third or fourth left intercostal space (Fig. 1B). The Doppler sample volume was positioned in the left atrium behind the posterior aortic wall. The mitral valve orifice was explored for a systolic regurgitant flow by slight angulation of the transducer and by moving the electronic sampling gate back and forth (Fig. 4). The first 37 patients were examined by both methods and the last 27 by the parasternal approach only.

\section{Results}

I: MITRAL STENOSIS

Correlations between Doppler flow patterns and haemodynamic data are shown in Fig. 5 and 6. Fig. 5 shows the results of 37 patients in sinus rhythm. All 14 patients with a smooth and bifid diastolic flow curve (Doppler pattern 0) had no mitral valve gradient. All patients with Doppler patterns I, II, and III had gradients and the severity of gradient could also be estimated from the flow pattern. In the 4 patients showing Doppler pattern I, the gradient was $5 \cdot 7 \pm 1 \cdot 5$ (SD). Doppler pattern II observed in 12 patients was associated with a mean gradient of $10 \cdot 3 \pm 3.7$ and pattern III in 7 patients with a gradient of $22.9 \pm 7 \cdot 9$. The difference between groups with patterns 0 and II and with patterns II and III were statistically significant. 


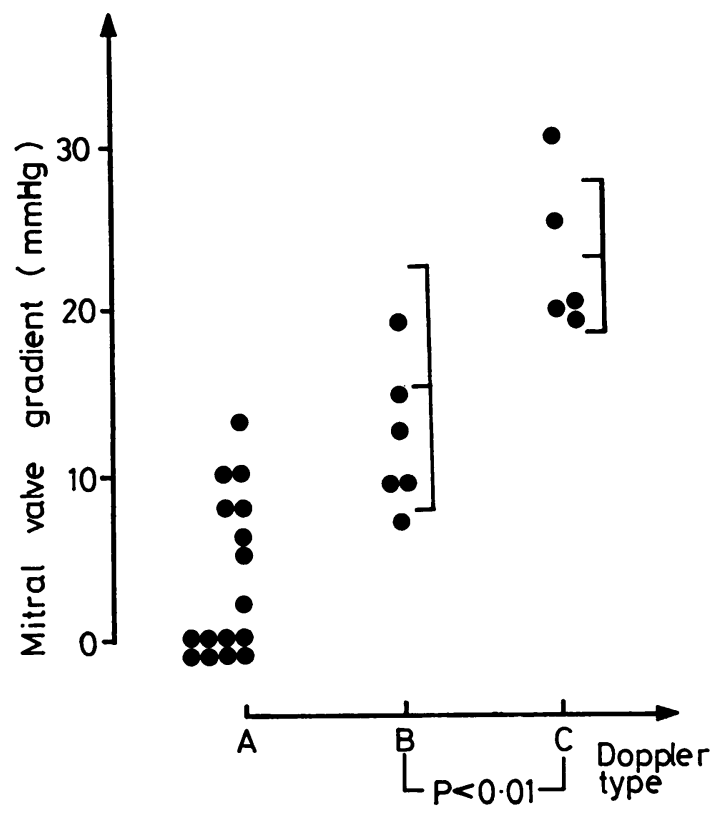

Fig. 6 Mean gradient plotted against the Doppler type of curve in patients with atrial fibrillation. Types $B$ and $C$ appear specific and allow an appreciation of the severity of mitral stenosis. In type $A$, the poor sensitivity indicates the actual limitation of this method.

Fig. 6 shows the results in patients with atrial fibrillation. Half of the 16 patients showing a flow ending before end-diastole (type A) had gradients. A flow with a dome-shaped appearance (type B) or ascending throughout long diastoles (type $C$ ) was associated in all patients with gradients (mean of $15 \pm 7.6 \mathrm{mmHg}$ and $22.8 \pm 4 \cdot 7$, respectively, $\mathrm{P}<$ 0.01 ).

The mean gradient in Doppler type B in atrial fibrillation was similar to the mean gradient of pattern II in sinus rhythm and the mean gradient of type C was similar to that of pattern III in sinus rhythm. The presence or the absence of associated mitral regurgitation did not alter the results.

\section{II: MITRAL REGURGITATION}

Results obtained with both methods of analysis of mitral regurgitation were compared in the first 37 patients and are shown in Table 1. Fig. 2 and 4 illustrate examples of recordings by each method. Of 13 patients without mitral regurgitation on angiography a transmitral systolic flow was recorded by the apical approach in 7 and by the parasternal approach in only 2 . Of 14 patients with mild mitral regurgitation (grade 1 on angiography), 2 could be identified by the apical approach and 9 by the parasternal approach. Of the 10 patients with moderate to severe mitral regurgitation (grades 2 and 3), 7 were found by the apical and 9 by the parasternal position. The specificity of the parasternal approach was 85 per cent compared with 46 per cent for the apical approach $(P<0.05)$. The sensitivity for the diagnosis of all degrees of mitral regurgitation was 75 per cent for the parasternal approach and 37 per cent for the apical approach $(\mathbf{P}<0.01)$. For moderate to severe regurgitation, sensitivity was 90 per cent when sampling in the left atrium versus 70 per cent by the apical approach (NS).

Because of its superiority, the parasternal approach was used exclusively in the 27 subsequent patients. As shown in Table 2, of the total of 64 patients, 20 had no angiographic mitral valve regurgitation and the Doppler diagnosis was correct in 18, yielding a specificity of 90 per cent. Overall sensitivity was 59 per cent. Considering only angiographic grade 2 and grade 3 mitral regurgita-

Table 1 Doppler analysis of mitral regurgitation: comparison of the apical and parasternal approaches

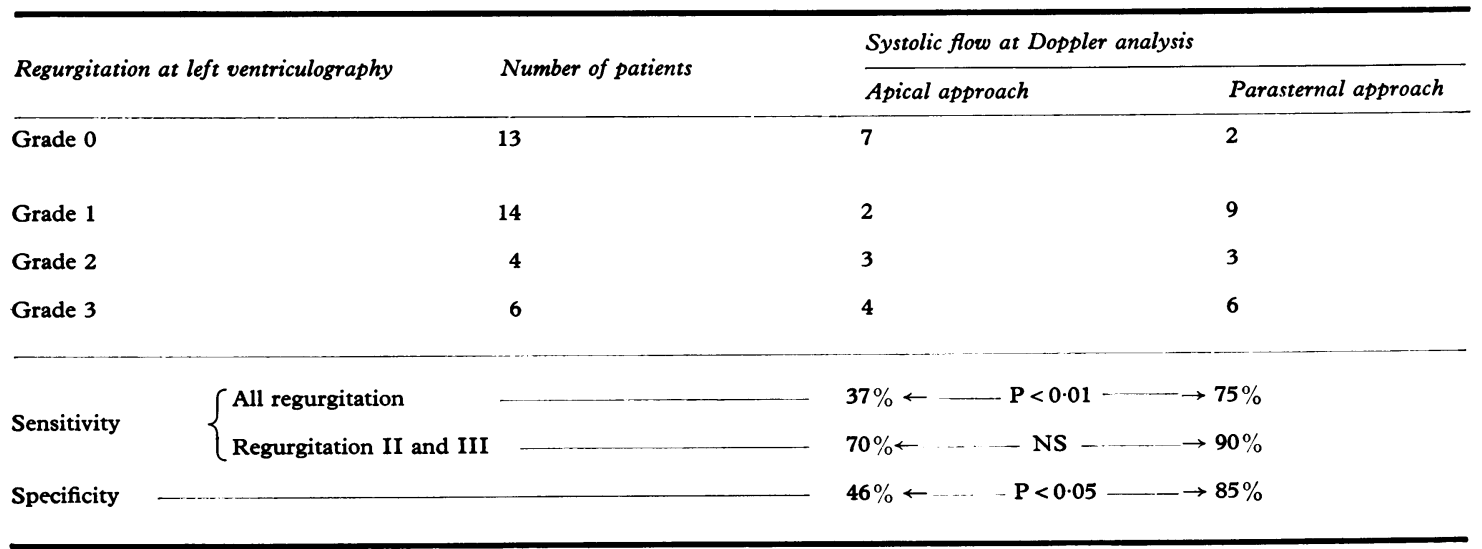


Table 2 Doppler analysis of mitral regurgitation: parasternal approach

\begin{tabular}{lcccc}
\hline \multirow{2}{*}{ Doppler } & \multicolumn{4}{l}{ Ventriculography regurgitation } \\
\cline { 2 - 5 } & Grade 0 & Grade 1 & Grade 2 & Grade 3 \\
\hline+ & 2 & 11 & 5 & 10 \\
- & 18 & 16 & 1 & 1 \\
\hline & |P $<0.0001 \star$ & &
\end{tabular}

Sensitivity $\left\{\begin{array}{l}\text { All regurgitation }-59 \% \\ \text { Regurgitation II and III }-88 \%\end{array}\right.$

Specificity -

+ Indicates the identification of a systolic flow in the left atrium.

- Indicates the absence of a systolic flow in the left atrium.

* The $P$ value was calculated by the Fisher probability test.

tion, the sensitivity increased to 88 per cent $(15 / 17)$.

\section{Discussion}

The applicability of pulsed Doppler echocardiography to the study of mitral valve lesions was first shown by Johnson et al., in 1973. More recently, Kalmanson et al. $(1975,1977)$ using transseptal Doppler ultrasound catheterisation and transcutaneous pulsed Doppler method described different patterns of flow curves which they related to the severity of mitral stenosis as assessed by clinical and haemodynamic studies. The present study correlates the results of external Doppler analysis to the severity of mitral valve stenosis and regurgitation as assessed in all patients by catheterisation.

\section{I: MITRAL STENOSIS}

The maximum Doppler shift that can be detected is governed by sampling theory concepts, that is the pulse repetition rate must be twice the highest expected Doppler shift. Since the pulse repetition rate has to be lower with increasing transcutaneous depth of the sample volume, it can be postulated that some of the high-velocity jets encountered in mitral stenosis could not be recorded deep in the thorax especially when the angle is close to zero. However, the recorded velocity represents the average of velocities in the sample volume that is the mean forward flow. The plus or minus flow caused by swirling and eddying are expected to neutralise in part because of the size of the sample volume and because of the motion of the heart within the chest wall. In none of the patients studied was the flow curve observed to saturate.
In sinus rhythm, Doppler analysis detected all cases of mitral stenosis and no false positive recordings were observed. Moreover, semiquantification was achieved and statistically significant differences were found according to the degree of stenosis. The flow curves in atrial fibrillation could not differentiate patients with mild mitral stenosis from those without a mitral valve gradient. However, patterns of flow in moderate and severe stenosis resembled patterns in sinus rhythm and the haemodynamic gradients were also comparable. The method in atrial fibrillation, if not accurate for the detection of mild mitral stenosis, appears useful for the semiquantification of the degree of stenosis. Though the mitral valve gradients of a few patients with each Doppler pattern overlap, the method appears promising as a clinical adjunct for assessing the severity of mitral stenosis. Further work is needed to refine the analysis of the flow curves in order to improve their diagnostic value.

The criteria of severity for mitral stenosis previously described (Kalmanson et al., 1975, 1977) included indentations on the flow curve, a decreased rate of rise of flow velocity, and a large $A$ wave. It was reported that the greater the severity of the stenosis the more obvious were these abnormalities. Our flow patterns were grossly similar but had notably different characteristics which were useful in assessing the degree of mitral stenosis. If an increased amplitude of the atrial flow wave was observed in mild mitral stenosis, this atrial wave disappeared with more severe degrees of stenosis. It was sometimes observed in moderate or severe stenosis but then seemed clearly related to the audio signal from the mitral valve leaflets. Slight adjustment of the sample volume restored a pure signal and the so-called atrial wave disappeared. Severe stenosis was associated with a quick ascending limb followed by a slowly ascending plateau. These differences from previously reported curves could be explained in several ways. First the site of the sample volume appears critical for the quality and the reproducibility of the curve and this was not emphasised in previous work. Ultrasounds reflected from moving structures in the left ventricle may introduce artefacts in the filling curves. Secondly, the derived analogue curve represents the mean of the velocities in the sample volume and the method of zero crossing analysis may differ between different apparatus. Thirdly, the difference in the characteristics of the filter used to suppress noise may alter the pattern of the flow curve.

\section{II: MITRAL REGURGITATION}

So far, pulsed Doppler evaluation of mitral regurgitation has been explored by 2 methods. The first, 
described by Johnson et al. (1973), consists of identifying a regurgitant flow in the left atrium with the transducer located in the parasternal position. More recently Stevenson et al. (1977) used this approach to differentiate mitral regurgitation from ventricular septal defect. However, these reports have included few correlations with the objective results of angiography. In our hands, this method was satisfactory in grades 2 and 3 mitral regurgitation; only 2 cases were not recorded and they both occurred at the beginning of the study when our experience was limited. Thus they might represent poor technique rather than true false negative results. In the future a sensitivity of 100 per cent is expected for the diagnosis of moderate to severe mitral regurgitation. The 2 false-positive recordings of a systolic flow deserve further comment. We feel that they might represent flow from the pulmonary veins. Indeed, we suspect that the double echo occasionally seen on the left atrium $A$ and $M$-mode echocardiogram may represent a pulmonary vein. Sampling in this area would yield a systolodiastolic flow. Careful siting of the electronic sampling gate could thus not only avoid recording this systolic flow but also characterise the nature of the double echo sometimes seen on the left atrial echocardiogram. More work is needed to define the value of the method in mild mitral regurgitation.

The other method of evaluating mitral regurgitation was described by Kalmanson et al. (1977) and consisted in identifying a systolic flow running away from the transducer positioned at the apex. In our study, the sensitivity and specificity of this method were significantly lower than with the parasternal approach. In particular, patterns of large mitral regurgitation were noted in the absence of angiographic reflux (Fig. 2). We think that the difficulties encountered with this approach are related to technical problems of lateral resolution with the apparatus we use. The sample volume overrides the inflow and outflow tracts of the left ventricle and a systolic flow corresponding to systolic ejection in the left ventricle may be confused with a true regurgitant flow through mitral valve. Hopefully, improvement in the pulsed Doppler technical characteristics will allow a more accurate evaluation of mitral regurgitation by this approach. Indeed, this method presents theoretical advantages in that the regurgitant flow is running in the axis of the ultrasound beam, optimising the Doppler shift of frequency. We expect that the development of a Doppler apparatus with improved signal-tonoise ratio will improve performance and also that the process for deriving the signal will be improved.
Indeed, the recorded signal is a relatively poor reflection of the information contained in the audio signal. Spectral analysis of the Doppler signal should allow more adequate characterisation of the transmitral valve flow and more accurate quantification of mitral regurgitation and stenosis.

In future studies, location of the Doppler sample volume should be carefully described as well as the characteristics of the instrumentation used, particularly with respect to its filter and to the process of analysing the signal.

This work was supported in part by the J. L. Lévesque Foundation and in part by a Grant from the CNAMTS (France).

\section{References}

Baker, D. W. (1970). Pulsed ultrasonic Doppler blood flow sensing. IEEE Transactions on Sonics and Ultrasonics, SU17, 170-195.

Feigenbaum, H. (1976). Echocardiography, pp. 107-113. Lea and Febiger, Philadelphia.

Gorlin, R., and Gorlin, S. G. (1951). Hydraulic formula for calculation of the area of the stenotic mitral valve, other cardiac valves and central circulation. American Heart fournal, 41, 1-29.

Henry, W. L., Griffith, J. M., Michaelis, L. L., McIntosh, C. L., Morrow, A. G., and Epstein, S. E. (1975). Measurement of mitral orifice area in patients with mitral valve disease by real-time, two-dimensional echocardiography. Circulation, 51, 827-831.

Johnson, S. L., Baker, D. W., Lute, R. A., and Dodge, H. T. (1973). Doppler echocardiography. The localization of cardiac murmurs. Circulation, 48, 810-822.

Kalmanson, D., Veyrat, C., Bernier, A., Savier, C. H., Chiche, P., and Witchitz, S. (1975). Diagnosis and evaluation of mitral valve disease using transseptal Doppler ultrasound catheterization. British Heart fournal, 37, 257271.

Kalmanson, D., Veyrat, C., Bouchareine, F., and Degroote, A. (1977). Non-invasive recording of mitral valve flow velocity patterns using pulsed Doppler echocardiography. British Heart fournal, 39, 517-528.

McLaughlin, M. J., and Morch, J. E. (1971). Cineangiography in mitral regurgitation. A comparison of cineangiography with other methods of assessment, particularly constant infusion of ${ }^{133} \mathrm{Xe}$. Investigative Radiology, 6, 416-425.

Péronneau, P., and Léger, F. (1969). Doppler ultrasonic pulsed blood flowmeter. In Proceedings of the Eighth International Conference on Medical and Biological Engineering, pp. 10-11. Chicago-IEEE Eds.

Stevenson, J. G., Kawabori, I., and Guntheroth, W. G. (1977). Differentiation of ventricular septal defects from mitral regurgitation by pulsed Doppler echocardiography. Circulation, 56, 14-18.

Requests for reprints to Dr Pierre Théroux, Montreal Heart Institute, 5000 East, Belanger Street, Montreal, Quebec H1T 1C8, Canada. 